\title{
THE GENERALIZED ZAHORSKI CLASS STRUCTURE OF SYMMETRIC DERIVATIVES
}

BY

LEE LARSON

\begin{abstract}
A generalized Zahorski class structure is demonstrated for symmetric derivatives. A monotonicity theorem is proved and a condition sufficient to ensure that a symmetric derivative has the Darboux property is presented.
\end{abstract}

1. Preliminaries. In $1950 \mathrm{Z}$. Zahorski [9] began a classification of the derivatives of continuous functions based upon the structure of their associated sets. He defined a descending sequence of subclasses of the Darboux-Baire one functions which form a stratification of the class of derivatives he considered. Kundu [6], in 1976, generalized the Zahorski classes in order to extend the theorems of Zahorski to include symmetric derivatives which have the Darboux property and continuous primitives. Our purpose in this work is to replace Kundu's requirement that the primitives be continuous by the more general requirement of measurability and, when possible, to remove the requirement that the symmetric derivatives have the Darboux property.

It will be assumed here that, unless it is specifically mentioned otherwise, all functions are finite valued and have their domains contained in $\mathbf{R}$, the real numbers. If $f$ is a function, then we denote

$$
C(f)=\{x: f \text { is continuous at } x\}
$$

and

$$
D(f)=\left\{x: f^{\prime}(x) \text { exists and is finite }\right\},
$$

where $f^{\prime}(x)$ is the ordinary derivative of $f$ at $x . \operatorname{If~}_{\lim _{h \rightarrow 0}}[f(x+h)-f(x-h)]=0$, then $f$ is symmetrically continuous at $x$. When

$$
\lim _{h \rightarrow 0} \frac{f(x+h)-f(x-h)}{2 h}
$$

exists, whether finite or infinite, then $f$ is symmetrically differentiable at $x$ and its symmetric derivative is denoted $f^{s}(x)$.

The complement of an arbitrary set $A \subset \mathbf{R}$ is denoted by $A^{\mathrm{c}}$ and its closure is $\bar{A}$. For simplicity we write $(\bar{A})^{\mathrm{c}}$ as $\bar{A}^{\mathrm{c}}$. If $A$ is measurable, then its measure is written as $|A|$.

Received by the editors October 25, 1982.

1980 Mathematics Subject Classification. Primary 26A24.

Key words and phrases. Symmetric derivative, Baire class one, Darboux property, Zahorski classes, nonangular. 
The class of all functions $f: \mathbf{R} \rightarrow \mathbf{R}$ such that $C(f)$ is dense and $f^{\mathrm{s}}$ exists everywhere is denoted by $m \Sigma$. We write $\mathfrak{B}_{1}$ for the functions of Baire class one and (D) for the functions with the Darboux property. The intersection of function classes is denoted by juxtaposition; e.g., $\mathscr{D} \cap \mathfrak{B}_{1}=\mathscr{D Q B}_{1}$.

Following Kundu [6], we present abstractions of the associated set definitions from Zahorski [9].

Let $A \subset \mathbf{R}$.

$M_{0}(A)$ is the collection of all $F_{\sigma}$ sets $F$ such that for all $x \in A \cap F, x$ is a bilateral limit point of $F$.

$M_{1}(A)$ is the collection of all $F_{\sigma}$ sets $F$ such that for all $x \in A \cap F, x$ is a bilateral condensation point of $F$.

$M_{2}(A)$ is the family of all $F_{\sigma}$ sets $F$ such that for all $x \in A \cap F$ and all $\delta>0$, $|(x-\delta, x) \cap F|>0$ and $|(x, x+\delta) \cap F|>0$.

$M_{3}(A)$ is the collection of all $F_{\sigma}$ sets $F$ such that if $x \in A \cap F$ and $\left\{I_{n}\right.$ : $n=1,2, \ldots\}$ is any sequence of closed intervals converging to $x$ such that $I_{n} \cap F=$ $\varnothing$ for all $n$, then $\lim _{n \rightarrow \infty}\left[\left|I_{n}\right| / d\left(x, I_{n}\right)\right]=0$, where $d\left(x, I_{n}\right)$ is the distance between $x$ and $I_{n}$.

$M_{4}(A)$ is the collection of all $F_{\sigma}$ sets $F$ such that there is a sequence of closed sets $F_{n}$ and a sequence of numbers $\left\{\eta_{n}\right\} \subset(0,1)$ such that $F=\cup_{n=1}^{\infty} F_{n}$, and for every $c>0$ and every $x \in A \cap F_{n}$ there is an $\varepsilon(x, c)>0$ such that for any two real numbers $h$ and $h_{1}$, satisfying $h h_{1}>0, c h_{1}>h$ and $\left|h+h_{1}\right|<\varepsilon(x, c)$, the relation $|F \cap J| /|J|>\eta_{n}$ is true, where $J$ is the interval with endpoints $x+h$ and $x+h+h_{1}$.

$M_{5}(A)$ is the collection of all $F_{\sigma}$ sets $F$ such that for all $x \in A \cap F, x$ is a density point of $F$.

For $i=0,1, \ldots, 5$, define the abstract Zahorski class $\mathscr{N}_{i}(A)$ to be the collection of all functions $f$ such that for any $a \in \mathbf{R},\{x: f(x)>a\}$ and $\{x: f(x)<a\}$ are both in $M_{i}(A)$.

Define the class $Z(A)$ to be the collection of all functions $f \in \mathfrak{B}_{1}$ such that for each $x \in A$, each $\varepsilon>0$ and each sequence of closed intervals $\left\{I_{n}\right\}$ converging to $x$ such that for each $n, f(y) \geqslant f(x)$ on $I_{n}$, or $f(y) \leqslant f(x)$ on $I_{n}$,

$$
\lim _{n \rightarrow \infty} \frac{\left|\left\{y \in I_{n}:|f(y)-f(x)| \geqslant \varepsilon\right\}\right|}{\left|I_{n}\right|+d\left(x, I_{n}\right)}=0 .
$$

(This is a pointwise characterization of Weil's class $Z$ [8].)

If $A=\mathbf{R}$ in any of the above definitions, then we omit the reference to $A$; e.g., $M_{3}(\mathbf{R})=M_{3}$. In this case, the classes mentioned above are the same as in the original definitions due to Zahorski or Weil, respectively.

LEMMA 1.1. If $A \subset \mathbf{R}$ is any set, then

$$
\mathfrak{\Re}_{0}(A) \supset \Re_{1}(A) \supset \Re_{2}(A) \supset \Re_{3}(A) \supset Z(A) \supset \Re_{4}(A) \supset \Re_{5}(A) .
$$

Proof. All of the inclusions in (1) except $Z(A) \subset \mathfrak{N}_{3}(A)$ are obvious from the definitions. To prove that $Z(A) \subset \mathfrak{R}_{3}(A)$, let $f \in Z(A)$ and $a \in \mathbf{R}$. It must be shown that the sets $B=\{x: f(x)>a\}$ and $C=\{x: f(x)<a\}$ are in $M_{3}(A)$. We will prove $B \in M_{3}(A)$; consideration of $-f$ then shows $C \in M_{3}(A)$. 
From the definition of $Z(A)$ we see $f \in \mathfrak{B}_{1}$, so $B$ is an $F_{\sigma}$ set. If $A \cap B=\varnothing$, it follows vacuously that $B \in M_{3}(A)$. So, suppose $A \cap B \neq \varnothing$ and choose an $x \in A$ $\cap B$. Let $\varepsilon=f(x)-a$ and choose a sequence of closed intervals, $\left\{I_{n}\right\}$, converging to $x$ such that $I_{n} \cap B=\varnothing$ for each $n$. Since $f \in Z(A)$ and $f(y) \leqslant f(x)$ for each $y \in I_{n}$ and each $n$, we see that

$$
\begin{aligned}
0 & =\lim _{n \rightarrow \infty} \frac{\left|\left\{y \in I_{n}:|f(y)-f(x)| \geqslant \varepsilon\right\}\right|}{\left|I_{n}\right|+d\left(x, I_{n}\right)} \\
& =\lim _{n \rightarrow \infty} \frac{\left|I_{n}\right|}{\left|I_{n}\right|+d\left(x, I_{n}\right)}=\lim _{n \rightarrow \infty}\left[1+\frac{d\left(x, I_{n}\right)}{\left|I_{n}\right|}\right]^{-1},
\end{aligned}
$$

from which it easily follows that

$$
\lim _{n \rightarrow \infty} \frac{\left|I_{n}\right|}{d\left(x, I_{n}\right)}=0
$$

and $f \in \mathscr{N}_{3}(A)$.

It will be useful to examine some properties of the functions in $m \Sigma$. For any $f \in m \Sigma$, define

$$
M_{f}=\left\{x:\left|\limsup _{\substack{t \rightarrow x \\ t \in C(f)}} f(t)\right|=\infty\right\}
$$

and

$$
\mu_{f}(x)= \begin{cases}\limsup _{t \rightarrow x} f(t), & x \notin M_{f}, \\ t \in C(f) & x \in M_{f} . \\ f(x), & \end{cases}
$$

In Larson [7] the following theorems are established. (See [7, Theorems 4.2 and 6.2].)

THEOREM 1.2. If $f \in m \Sigma$ then $\mu_{f} \in \mathfrak{B}_{1}$ and the following conditions are satisfied:

(a) $\bar{M}_{f}$ is countable;

(b) $\mu_{f}(x)=\lim \sup _{t \rightarrow x(+o r-)} \mu_{f}(t)$ when $x \in \bar{M}_{f}^{\mathrm{c}}$ and $f^{\mathrm{s}}(x)$ is finite;

(c) $C(f) \subset C\left(\mu_{f}\right)\left(D(f) \subset D\left(\mu_{f}\right)\right)$ and $f=\mu_{f}\left(f^{\prime}=\mu_{f}^{\prime}\right)$ on $C(f)(D(f))$; and

(d) $\mu_{f}^{\mathrm{s}}$ exists and equals $f^{\mathrm{s}}$ on $\bar{M}_{f}^{\mathrm{c}}$.

THEOREM 1.3. If $f \in m \Sigma$ such that $f^{\mathrm{s}} \geqslant 0$ a.e. and $f^{\mathrm{s}}>-\infty$ everywhere, then $\mu_{f}$ is nondecreasing. If, in addition, $f^{\mathrm{s}}<\infty$ everywhere, then $\mu_{f}$ is continuous.

Using these theorems we can establish the following theorem, which is similar to Theorem 3.1 of [7].

THEOREM 1.4. Let $f$ be symmetrically differentiable everywhere. The following statements are equivalent:

(a) $f$ is measurable;

(b) $D(f)$ has full measure; and,

(c) $f \in m \Sigma$. 
Proof. That (a) is equivalent to (b) is due to Khintchine [5, p. 217]. (b) implies (c) is obvious. To show that (c) implies (b), let $I$ be a component of $\bar{M}_{f}^{\mathrm{c}}$. Then $\mu_{f}$ is symmetrically differentiable and measurable on $I$ according to Theorem 1.2. Using Khintchine's theorem again, we see that $\left|\mu_{f}^{\mathrm{s}}\right|<\infty$ a.e. on $I$. According to Belna, Evans and Humke [1, Theorem 2] there exists a set $A \subset I$ of full measure such that when $x \in A$, then

$$
f^{\mathrm{s}}(x)=\min \left\{D_{+} f(x), D_{-} f(x)\right\}=\max \left\{D^{+} f(x), D^{-} f(x)\right\} .
$$

An application of the Denjoy-Young-Saks Theorem [2, p. 65] yields that $D(f)$ has full measure on $I$. Applying this argument to each component of $\bar{M}_{f}^{\mathrm{c}}$ and noting that $\bar{M}_{f}^{c}$ is countable by Theorem 1.2(a), we see that $D(f)$ has full measure on $\mathbf{R}$, which is (b).

K. Garg [4] has called a function $f$ nonangular at $x$ if

$$
D^{+} f(x) \geqslant D_{-} f(x) \text { and } D^{-} f(x) \geqslant D_{+} f(x) .
$$

If (2) fails to hold, then $f$ is angular at $x$. In particular, if $f$ is differentiable at $x$, then it is nonangular at $x$. In general, it is not difficult to show that an arbitrary function can be angular on at most a countable set. (See, e.g., Bruckner [2, p. 63].) But, symmetrically differentiable functions can be angular. For example, consider $f(x)=$ $|x|$ in which case $f^{\mathrm{s}}(0)=0$, but $f$ is angular at 0 . Also notice that in this case $f \in m \Sigma$, but $f^{\mathrm{s}} \notin \mathscr{Q}$. Observations such as this motivate the following theorem.

THEOREM 1.5. If $f \in m \Sigma$ such that $f$ is nonangular and symmetrically continuous everywhere, then $f^{s} \in \mathscr{Q}_{\mathcal{B}}$.

Proof. Since Theorem 2.1 of [7] ensures that $f^{\mathrm{s}} \in \mathcal{H}_{1}$, it suffices to show (Bruckner [2, p. 9]) that for each $x \in \mathbf{R}$ the following inclusion is true:

$$
\begin{aligned}
f^{\mathrm{s}}(x) \in & {\left[\liminf _{t \rightarrow x^{-}} f^{\mathrm{s}}(t), \limsup _{t \rightarrow x^{-}} f^{\mathrm{s}}(t)\right] } \\
& \cap\left[\liminf _{t \rightarrow x^{+}} f^{\mathrm{s}}(t), \limsup _{t \rightarrow x^{+}} f^{\mathrm{s}}(t)\right] .
\end{aligned}
$$

Suppose (3) fails to hold at $x_{0}$; e.g., suppose

$$
f^{\mathrm{s}}\left(x_{0}\right)<\liminf _{t \rightarrow x_{0}+} f^{\mathrm{s}}(t)
$$

Through the addition of an appropriate linear term to $f$ it may be supposed without loss of generality that

$$
f^{\mathrm{s}}\left(x_{0}\right)<0<\liminf _{t \rightarrow x_{0}+} f^{\mathrm{s}}(t) .
$$

This implies there is an $\varepsilon>0$ such that $f^{\mathrm{s}}(t)>0$ whenever $t \in\left(x_{0}, x_{0}+\varepsilon\right)$. It is clear that the nonangularity of $f$ implies that for each $x \in \mathbf{R}, f$ must satisfy

$$
\liminf _{t \rightarrow x} f(t) \leqslant f(x) \leqslant \limsup _{t \rightarrow x} f(t) .
$$

Therefore, according to Evans [3], $f$ is strictly increasing on $\left(x_{0}, x_{0}+\varepsilon\right)$.

Suppose

$$
f\left(x_{0}\right)>\lim _{t \rightarrow x_{0}+} f(t),
$$


where the limit on the right exists because $f$ is monotone on $\left(x_{0}, x_{0}+\varepsilon\right)$. Then the symmetric continuity of $f$ implies

$$
f\left(x_{0}\right)>\limsup _{t \rightarrow x_{0}-} f(t) .
$$

From (6) and (7) we see that $D^{+} f\left(x_{0}\right)=-\infty$ and $D_{-} f\left(x_{0}\right)=\infty$, which violates (2). Therefore, $f\left(x_{0}\right) \leqslant \lim _{t \rightarrow x_{0}} f(t)$. In a similar manner it follows that $f\left(x_{0}\right) \geqslant$ $\lim _{t \rightarrow x_{0}}+f(t)$. Combining these two inequalities we see that $f\left(x_{0}\right)=\lim _{t \rightarrow x_{0}}+f(t)$. Because $f$ is increasing on $\left(x_{0}, x_{0}+\varepsilon\right)$ it is now evident that

$$
D_{+} f\left(x_{0}\right) \geqslant 0 \text {. }
$$

Since $f^{\mathrm{s}}\left(x_{0}\right)<0$ and $f(t)>f\left(x_{0}\right)$ on $\left(x_{0}, x_{0}+\varepsilon\right)$ there is a $\delta>0$ such that $f(t)>f\left(x_{0}\right)$ on $\left(x_{0}-\delta, x_{0}\right)$. Therefore,

$$
D^{-} f\left(x_{0}\right) \leqslant 0 \text {. }
$$

Inequalities (2), (8) and (9) imply that

$$
D_{+} f\left(x_{0}\right)=D^{-} f\left(x_{0}\right)=0 .
$$

It follows from (10) that there is an increasing sequence $x_{n} \rightarrow x_{0}$ such that

$$
\lim _{n \rightarrow \infty} \frac{f\left(x_{0}\right)-f\left(x_{n}\right)}{x_{0}-x_{n}}=0
$$

and (8) implies

$$
\liminf _{n \rightarrow \infty} \frac{f\left(2 x_{0}-x_{n}\right)-f\left(x_{0}\right)}{x_{0}-x_{n}} \geqslant 0 .
$$

Consider

$$
\begin{aligned}
f^{\mathrm{s}}\left(x_{0}\right) & =\lim _{n \rightarrow \infty} \frac{f\left(2 x_{0}-x_{n}\right)-f\left(x_{n}\right)}{2\left(x_{0}-x_{n}\right)} \\
& =\lim _{n \rightarrow \infty}\left[\frac{f\left(2 x_{0}-x_{n}\right)-f\left(x_{0}\right)}{2\left(x_{0}-x_{n}\right)}+\frac{f\left(x_{0}\right)-f\left(x_{n}\right)}{2\left(x_{0}-x_{n}\right)}\right] \\
& =\liminf _{n \rightarrow \infty} \frac{f\left(2 x_{0}-x_{n}\right)-f\left(x_{0}\right)}{2\left(x_{0}-x_{n}\right)}+\lim _{n \rightarrow \infty} \frac{f\left(x_{0}\right)-f\left(x_{n}\right)}{2\left(x_{0}-x_{n}\right)} \\
& \geqslant 0 .
\end{aligned}
$$

This is a contradiction of (5), so we are forced to conclude that (4) never occurs.

The impossibility of the other assumptions which violate (3) is established similarly.

Perhaps it is worth noting that the converse to Theorem 1.5 is false. To see this, consider

$$
f(x)= \begin{cases}\frac{x}{3} \sin \frac{1}{x}+x, & x<0 \\ 0, & x=0 \\ \frac{x}{3} \sin \frac{1}{x}, & x>0 .\end{cases}
$$


It is easy to show that $f \in m \Sigma$ and $f^{\mathrm{s}} \in \mathscr{D} \mathcal{B}_{1}$, but $D^{+} f(0)=\frac{1}{3}<D_{-} f(0)=\frac{2}{3}$ violates (2).

2. Symmetric derivatives and the class $\mathscr{T}_{2}$. The main goal of this section is to prove the following theorem.

THEOREM 2.1. If $f \in m \Sigma$ such that $f^{\mathrm{s}} \in \mathcal{O}$, then $f^{\mathrm{s}} \in \mathfrak{M}_{2}$.

To prove this theorem we first prove the following lemma.

LEMMA 2.2. Let $f \in m \Sigma$ such that $f^{\mathrm{s}} \in \mathcal{O}$. If $f^{\mathrm{s}} \geqslant 0$ a.e., then $\mu_{f}$ is nondecreasing.

Proof. According to Theorem 1.3 it suffices to show that $A=\left\{x: f^{\mathrm{s}}(x)=-\infty\right\}$ is empty. Define $B=\left\{x: f^{\mathrm{s}}(x) \leqslant-1\right\}$ and $C=\left\{x: f^{\mathrm{s}}(x)=-2\right\}$. We claim that $A$ is relatively dense in $B$. To see this, suppose it is not. Then there is an open interval $I$ such that $I \cap A=\varnothing$ and $I \cap B \neq \varnothing$. Theorem 1.3 implies $\mu_{f}$ is nondecreasing on $I$. Let $x \in I$. Since $C(f)$ is a dense $G_{\delta}$ set, it is residual, so there exists a sequence $\left\{x_{n}\right\} \subset C(f)$ such that $2 x-x_{n} \in C(f)$ for each $n$ and $x_{n}$ increases to $x$. Using Theorem 1.2(c), it follows that

$$
f^{\mathrm{s}}(x)=\lim _{n \rightarrow \infty} \frac{f\left(2 x-x_{n}\right)-f\left(x_{n}\right)}{2\left(x-x_{n}\right)}=\lim _{n \rightarrow \infty} \frac{\mu_{f}\left(2 x-x_{n}\right)-\mu_{f}\left(x_{n}\right)}{2\left(x-x_{n}\right)} \geqslant 0 .
$$

This implies $I \cap B=\varnothing$, which is a contradiction of the choice of $I$. Therefore $A$ is relatively dense in $B$.

We now claim $C$ is also relatively dense in $B$. To see this, let $x \in B$. Since $A$ is relatively dense in $B$, we may choose a sequence $\left\{x_{n}\right\} \subset A$ such that $x_{n} \rightarrow x$. By assumption, the set $\left\{x: f^{\mathrm{s}}(x) \geqslant 0\right\}$ is dense in $\mathbf{R}$, so for each $n$ there is a $y_{n}$ such that $f^{\mathrm{s}}\left(y_{n}\right) \geqslant 0$ and $\left|x_{n}-y_{n}\right|<1 / n$. Finally, $f^{\mathrm{s}} \in \mathcal{D}$ allows us to choose a $z_{n}$ between $x_{n}$ and $y_{n}$ such that $f^{\mathrm{s}}\left(z_{n}\right)=-2$. It is clear that $\left\{z_{n}\right\} \subset B \cap C$ and $z_{n} \rightarrow x$. Therefore, $C$ is relatively dense in $B$.

From Theorem 2.1 of [7] it follows that $A, B$ and $C$ are each $G_{\delta}$ sets. According to the above arguments, $A$ and $C$ are disjoint residual subsets of $B$, which is a Baire space. This is a contradiction and forces us to conclude that $A=\varnothing$.

In order to prove Theorem 2.1 , let $f$ be as in the theorem and let $A=\{x$ : $\left.f^{\mathrm{s}}(x)>0\right\}$. Suppose there is an $x \in A$ and an $\varepsilon>0$ such that

$$
|(x, x+\varepsilon) \cap A|=0 .
$$

Then $f^{\mathrm{s}} \leqslant 0$ a.e. on $(x, x+\varepsilon)$. Using $-f$ in Lemma 1.2 it follows that $f^{\mathrm{s}} \leqslant 0$ everywhere on $(x, x+\varepsilon)$. Since $f^{\mathrm{s}}(x)>0$, this is clearly a contradiction of $f^{\mathrm{s}} \in \mathscr{O}$.

In a similar manner, it can be shown that $|(x-\varepsilon, x) \cap A|>0$ for each $x \in A$ and $\varepsilon>0$. Therefore $A \in \mathfrak{K}_{2}$.

If $A=\left\{x: f^{\mathrm{s}}(x)<0\right\}$, then consider $-f$ with the preceding argument. If $A=\{x$ : $\left.f^{\mathrm{s}}(x)>c\right\}$ or $A=\left\{x: f^{\mathrm{s}}(x)<c\right\}$, then use $g(x)=f(x)-c x$ to reduce the problem to one of the preceding cases.

The next corollary follows immediately from Theorems 1.5 and 2.1.

COROLlARY 2.3. If $f \in m \Sigma$ such that $f$ is nonangular and symmetrically continuous, then $f^{\mathrm{s}} \in \mathscr{N}_{2}$. 
Theorem 2.1 generalizes Zahorski's original theorem [9, p. 21]. It also improves upon the result of Kundu [6] which required $f$ to be continuous and $f^{\mathrm{s}}$ to be in $\mathscr{D}$ and finite on a cocountable set. Since it was shown by Zahorski [9] that $\mathscr{T}_{2} \subset \mathscr{D}_{B_{1}}$ and that there exists an ordinary derivative which is not in $\Re_{3}$, we see that Theorem 2.1 cannot be substantially strengthened.

3. Symmetric derivatives and the class $\Re_{3}$. Zahorski $[9$, p. 21] showed that any finite derivative is in $\mathfrak{K}_{3}$. From this and Theorem 2.1, one might conjecture that any $f \in m \Sigma$ such that $f^{s}$ is everywhere-finite and $f^{s} \in \mathscr{D}$ is in $\mathscr{K}_{3}$. Unfortunately, the situation is a bit more complicated, as can be seen from the following example.

EXAMPLE. There is a continuous and nonangular $f \in m \Sigma$ such that $f^{\mathrm{s}} \in \mathscr{Q} \mathfrak{B}_{1}$ and $\left|f^{\mathrm{s}}(x)\right| \leqslant 3$ for all $x$, but $f^{\mathrm{s}} \notin \mathfrak{M}_{3}$.

For each positive integer $n$, let $I_{n}=\left(3^{-n-1}, 3^{-n}\right]$. If $x \in I_{n}$, then define

$$
\begin{aligned}
r_{n}(x)= & 2\left(x-\frac{1}{3^{n+1}}\right), \quad x \in\left(\frac{1}{3^{n+1}}, \frac{2}{3^{n+1}-3^{2 n+1}}\right), \\
- & \frac{3^{2 n+1}}{2}\left(x-\frac{2}{3^{n+1}}\right)^{2}+\frac{2}{3^{n+1}}-\frac{2}{3^{2 n+1}}, \\
& \quad x \in\left[\frac{2}{3^{n+1}-3^{2 n+1}}, \frac{2}{3^{n+1}+3^{2 n+1}}\right], \\
= & -2\left(x-\frac{1}{3^{n}}\right), \quad x \in\left(\frac{2}{3^{n+1}+3^{2 n+1}}, \frac{1}{3^{n}}\right] .
\end{aligned}
$$

Using these functions, define a function $f$ with domain $\mathbf{R}$ by

$$
f(x)= \begin{cases}(-1)^{n} r_{n}(x), & x \in I_{n}, \\ 0, & x=0 \text { or }|x|>\frac{1}{3}, \\ (-1)^{n} r_{n}(-x)-x, & -x \in I_{n} .\end{cases}
$$

It is an easy calculation to show that $f$ is differentiable on $\mathbf{R}-\{0\}$ with $\left|f^{\prime}(x)\right| \leqslant 3$ whenever $x \neq 0$. It is also evident from the symmetry of its definition that $f^{\mathrm{s}}(0)=\frac{1}{2}$. Therefore, $f \in m \Sigma$.

For each $n, r_{n}$ attains its maximum value on $I_{n}$ at $2 /\left(3^{n+1}\right)$. Thus,

$$
D^{+} f(0)=\lim _{n \rightarrow \infty} \frac{3^{2 n+1}}{2} r_{2 n}\left(\frac{2}{3^{2 n-1}}\right)=\lim _{n \rightarrow \infty}\left(1-3^{-2 n}\right)=1 \text {. }
$$

Similarly, $D_{+} f(0)=-1$. From this and the definition of $f$ it follows that $D^{-} f(0)=0$ and $D_{-} f(0)=-2$. Thus, $f$ satisfies (2) at $x=0$. It satisfies (2) everywhere else because it is differentiable away from $x=0$. Therefore, $f$ is nonangular. It is clear that $f$ is continuous, so Theorem 1.5 implies $f^{s} \in \mathscr{D} \mathfrak{B}_{1}$.

Let $A=\left\{x: f^{\mathrm{s}}(x)>0\right\}$. Since $f^{\mathrm{s}}(0)=\frac{1}{2}$, we see $0 \in A$. From the definition of $r_{n}(x)$, it is clear that $r_{n}^{\prime}(x) \leqslant 0$ whenever $x \in J_{n}=\left[2 /\left(3^{n+1}\right), 1 / 3^{n}\right]$. Observe that $J_{2 n} \cap A=\varnothing$ for all $n$ and $J_{n} \rightarrow 0$. Since

$$
\lim _{n \rightarrow \infty} \frac{\left|J_{2 n}\right|}{d\left(0, J_{2 n}\right)}=\lim _{n \rightarrow \infty} \frac{3^{-2 n-1}}{2 \cdot 3^{-2 n-1}}=\frac{1}{2}
$$

we conclude $f \notin \mathscr{N}_{3}$. 
Notice that this example also invalidates the next natural assumption that a bounded symmetric derivative with the Darboux property is in $\mathfrak{M}_{4}$.

The following theorem somewhat clarifies the situation.

THEOREM 3.1. If $f \in m \Sigma$, then $f^{\mathrm{s}} \in Z\left(D\left(\mu_{f}\right)\right)$.

Proof. Let $x_{0} \in D\left(\mu_{f}\right)$. Since $\mu_{f}^{\prime}\left(x_{0}\right)$ is finite by the definition of $D\left(\mu_{f}\right)$, through the addition of a linear function it may be assumed that

$$
f^{s}\left(x_{0}\right)=\mu_{f}^{\prime}\left(x_{0}\right)=\mu_{f}\left(x_{0}\right)=0 .
$$

Because $x_{0} \in D\left(\mu_{f}\right) \subset C\left(\mu_{f}\right)$, there is a $\delta>0$ such that $\left|\mu_{f}(t)\right|<1$ whenever $\left|t-x_{0}\right|<\delta$. Let $[a, b] \subset\left(x_{0}, x_{0}+\delta\right)$ be such that $f^{\mathrm{s}}(t) \geqslant 0$ on $[a, b]$ and let $\varepsilon>0$. Define

$$
A=\left\{t \in[a, b]: f^{\mathrm{s}}(t) \geqslant \varepsilon\right\} .
$$

We claim that

$$
\varepsilon|A| \leqslant \lim _{t \rightarrow b-} \mu_{f}(t)-\lim _{t \rightarrow a+} \mu_{f}(t) .
$$

To see this, first note that the limits in (12) make sense because Theorem 1.3 guarantees $\mu_{f}$ is nondecreasing on $(a, b)$. Define

$$
F_{n}(t)=n\left(\mu_{f}(t+1 / n)-\mu_{f}(t)\right) .
$$

It is clear that if $y \in D\left(\mu_{f}\right)$, then $F_{n}(y) \rightarrow \mu_{f}(y)$, so Theorem 1.2(c) and Khintchine [5, p. 217] imply $F_{n} \rightarrow f^{\text {s }}$ a.e.. Choose any interval $[c, d] \subset(a, b)$ such that $\{c, d\} \subset$ $C\left(\mu_{f}\right)$. Since $f^{\mathrm{s}}$ is nonnegative and measurable on $[c, d]$,

$$
\begin{aligned}
\varepsilon \mid\{x \in & {\left.[c, d]: f^{s}(x) \geqslant \varepsilon\right\} \mid \leqslant \int_{c}^{d} \liminf _{n \rightarrow \infty} F_{n}(t) d t } \\
& \leqslant \liminf _{n \rightarrow \infty} \int_{c}^{d} F_{n}(t) d t \\
& \leqslant \lim _{n \rightarrow \infty} \int_{d}^{d+1 / n} n \mu_{f}(t) d t-\lim _{n \rightarrow \infty} \int_{c}^{c+1 / n} n \mu_{f}(t) d t \\
& =\mu_{f}(d)-\mu_{f}(c)
\end{aligned}
$$

because $\mu_{f}$ is continuous at $c$ and $d$. Now, choose two sequences, $\left\{c_{n}\right\}$ and $\left\{d_{n}\right\}$, contained in $C\left(\mu_{f}\right)$, such that $c_{n}$ decreases to $a$ and $d_{n}$ increases to $b$. Then

$$
\begin{aligned}
\varepsilon|A| & =\lim _{n \rightarrow \infty} \varepsilon\left|\left\{x \in\left[c_{n}, d_{n}\right]: f(x) \geqslant \varepsilon\right\}\right| \\
& \leqslant \limsup _{n \rightarrow \infty}\left(\mu_{f}\left(d_{n}\right)-\mu_{f}\left(c_{n}\right)\right) \leqslant \lim _{t \rightarrow b-} \mu_{f}(t)-\lim _{t \rightarrow a+} \mu_{f}(t),
\end{aligned}
$$

which is (12).

Since $x_{0} \in D\left(\mu_{f}\right)$ and $\mu_{f}^{\prime}\left(x_{0}\right)=0$, we see that given an $r>0$ there is an $s \in(0, \delta)$ such that $0<t-x_{0}<s$ implies

$$
\left|\mu_{f}(t)-\mu_{f}\left(x_{0}\right)\right| \leqslant\left|t-x_{0}\right| \varepsilon r .
$$


Therefore, if $[a, b] \subset\left(x_{0}, x_{0}+s\right)$, then

$$
\begin{aligned}
& \left|\lim _{t \rightarrow b-} \mu_{f}(t)-\lim _{t \rightarrow a+} \mu_{f}(t)\right| \\
& \quad \leqslant\left|\lim _{t \rightarrow b-}\left(\mu_{f}(t)-\mu_{f}\left(x_{0}\right)\right)\right|+\left|\lim _{t \rightarrow a+}\left(\mu_{f}(t)-\mu_{f}\left(x_{0}\right)\right)\right| \\
& \quad \leqslant \lim _{t \rightarrow b-} \varepsilon r\left|t-x_{0}\right|+\lim _{t \rightarrow a+} \varepsilon r\left|t-x_{0}\right| \\
& \quad=\varepsilon r\left|b-x_{0}\right|+\varepsilon r\left|a-x_{0}\right|=\varepsilon r\left(2\left(a-x_{0}\right)+(b-a)\right) \\
& \quad \leqslant 2 \varepsilon r\left(d\left(x_{0},[a, b]\right)+|[a, b]|\right) .
\end{aligned}
$$

Combining this with (12), we see that

$$
|A| \leqslant 2 r\left(d\left(x_{0},[a, b]\right)+|[a, b]|\right) .
$$

In a similar manner, (14) can be established if $f^{\mathrm{s}}(t) \leqslant f^{\mathrm{s}}\left(x_{0}\right)$ for all $t \in[a, b]$, or if $[a, b] \subset\left(x_{0}-\delta, x_{0}\right)$. Since $r$ can be chosen arbitrarily, the theorem follows.

The following corollaries are immediate from Lemma 1.1, Theorem 1.2(c) and the definitions of $Z(A)$ and $\mathfrak{R}_{3}(A)$.

Corollary 3.2. If $f \in m \Sigma$, then $f^{\mathrm{s}} \in \Re_{3}\left(D\left(\mu_{f}\right)\right)$.

COROLlary 3.3. If $f \in m \Sigma$, then $f^{\mathrm{s}} \in Z(D(f))$.

Corollary 3.4. If $f \in m \Sigma$, then $f^{s} \in \mathfrak{N}_{3}(D(f))$.

Theorem 3.1 is a generalization of Weil's original theorem [8], that an everywhere-finite derivative is in $Z$. Corollary 3.4 is Kundu's original theorem without the requirements that $f$ be continuous and $f^{s} \in \mathcal{D}$.

4. Symmetric derivatives and the class $\mathfrak{N}_{4}$. The goal of this section is to prove the following theorem.

THEOREM 4.1. If $f \in m \Sigma$ such that $f^{\mathrm{s}}$ is bounded, then $f^{\mathrm{s}} \in \Re_{4}\left(D\left(\mu_{f}\right)\right)$.

The proof is immediate from the following two lemmas.

LEMMA 4.2. If $f \in m \Sigma,(a, b) \subset \mathbf{R}$ and $M \geqslant 0$ such that $f^{s} \leqslant M$ a.e., then

$$
\mu_{f}(b)-\mu_{f}(a) \leqslant M\left|\left\{x \in(a, b): f^{s}(x)>0\right\}\right| .
$$

Proof. For each positive integer $n$ define $F_{n}(x)$ as in (13). Theorem 1.3 implies $\mu_{f}$ is continuous, so each $F_{n}$ is also continuous. As before, $F_{n} \rightarrow f^{\text {s }}$ a.e.. Note that

$$
\begin{aligned}
\liminf _{n \rightarrow \infty} & \int_{a}^{b} F_{n}(x) d x=\liminf _{n \rightarrow \infty} n \int_{a}^{b}\left(\mu_{f}\left(x+\frac{1}{n}\right)-\mu_{f}(x)\right) d x \\
& =\liminf _{n \rightarrow \infty} n\left(\int_{b}^{b+1 / n} \mu_{f}(x) d x-\int_{a}^{a+1 / n} \mu_{f}(x) d x\right) \\
& \geqslant \liminf _{n \rightarrow \infty} n \int_{b}^{b+1 / n} \mu_{f}(x) d x-\limsup _{n \rightarrow \infty} n \int_{a}^{a+1 / n} \mu_{f}(x) d x \\
& =\mu_{f}(b)-\mu_{f}(a)
\end{aligned}
$$

because $\mu_{f}$ is continuous. 
Since $\mu_{f}$ is continuous, it is also clear that $M_{f}=\varnothing$ and, consequently, Theorem 1.2(d) implies $\mu_{f} \in m \Sigma$. Using this, the quasi-mean value theorem (Evans [3] or Larson [7, Theorem 7.1]) and (13) imply $F_{n}(x) \leqslant M$ for all $n$ and $x$. For each $n$ define

$$
G_{n}(x)=\max \left(F_{n}(x), 0\right) .
$$

Then $G_{n}$ is continuous for each $n$ with $0 \leqslant G_{n}(x) \leqslant M$ and $G_{n}(x) \rightarrow \max \left(f^{\mathrm{s}}(x), 0\right)$ a.e.. Applying the dominated convergence theorem yields

$$
\begin{aligned}
\liminf _{n \rightarrow \infty} & \int_{a}^{b} F_{n}(x) d x \leqslant \lim _{n \rightarrow \infty} \int_{a}^{b} G_{n}(x) d x \\
& =\int_{\left\{x \in(a, b): f^{s}(x)>0\right\}} f^{s}(x) d x \leqslant M\left|\left\{x \in(a, b): f^{s}(x) \geqslant 0\right\}\right| .
\end{aligned}
$$

A combination of (15) and (16) yields the lemma.

Lemma 4.3. If $f \in m \Sigma$ such that $f^{\mathrm{s}}$ is bounded above and $\alpha \in \mathbf{R}$, then

$$
E=\left\{x: f^{s}(x)>\alpha\right\} \in M_{4}\left(D\left(\mu_{f}\right)\right) .
$$

Proof. According to Larson [7, Theorem 2.1], $E$ is an $F_{\sigma}$ set. With no loss of generality it may be assumed that $\alpha=0$. Let $x_{0} \in E \cap D\left(\mu_{f}\right)$ and $\mu_{f}\left(x_{0}\right)=a$. Since $x_{0} \in D\left(\mu_{f}\right)$, for $h$ sufficiently close to 0 , we may write

$$
\mu_{f}\left(x_{0}+h\right)=\mu_{f}\left(x_{0}\right)+a h+h \eta(h)
$$

where $\eta(h) \rightarrow 0$ as $h \rightarrow 0$. If $h h_{1}>0$ and $\left|h+h_{1}\right|$ is sufficiently small, then

$$
\frac{\mu_{f}\left(x_{0}+h+h_{1}\right)-\mu_{f}\left(x_{0}+h\right)}{h_{1}}=a+\frac{h\left(\eta\left(h+h_{1}\right)-\eta(h)\right)}{h_{1}}+\eta\left(h+h_{1}\right) .
$$

Choose $c>0$ with $0<h / h_{1}<c$. For $h$ small enough, say $|h|<\varepsilon\left(x_{0}, c\right)$, (17) implies

$$
|\eta(h)|<a /(4 c+2) \text {. }
$$

Then, $\left|h+h_{1}\right|<\varepsilon\left(x_{0}, c\right)$ implies

$$
0<\frac{a}{2}<\frac{\mu_{f}\left(x_{0}+h+h_{1}\right)-\mu_{f}\left(x_{0}+h\right)}{h_{1}} .
$$

Now, let $M>1$ be an upper bound for $f^{\text {s }}$. Lemma 4.2 implies

$$
\begin{aligned}
& \frac{|h|}{h}\left(\mu_{f}\left(x_{0}+h+h_{1}\right)-\mu_{f}\left(x_{0}+h\right)\right) \\
& \quad \leqslant M\left|\left\{x \in\left(x_{0}+h, x_{0}+h+h_{1}\right): f^{\mathrm{s}}(x)>0\right\}\right| .
\end{aligned}
$$

It follows from (18) and (19) that

$$
\frac{\left|\left\{x \in\left(x_{0}+h, x_{0}+h+h_{1}\right): f^{s}(x)>0\right\}\right|}{\left|h_{1}\right|}>\frac{a}{2 M}>0 .
$$


For each positive integer $n$, the set $\left\{x: f^{\mathrm{s}}(x)>1 / n\right\}$ is an $F_{\sigma}$ set, so we may choose for each such $n$ a sequence of closed sets $\left\{E_{n, m}\right\}$ such that $\left\{x: f^{\varsigma}(x)>1 / n\right\}$ $=\cup_{m=1}^{\infty} E_{n, m}$. It is clear that

$$
E=\bigcup_{n=1}^{\infty}\left\{x: f^{\mathrm{s}}(x)>\frac{1}{n}\right\}=\bigcup_{n=1}^{\infty} \bigcup_{m=1}^{\infty} E_{n, m} .
$$

Since $x_{0} \in E$ there are integers $n$ and $m$ such that $x_{0} \in E_{n, m}$. Then $a>1 / n$ and from (20) we see

$$
\frac{\left|\left\{x \in\left(x_{0}+h, x_{0}+h+h_{1}\right): f^{\mathrm{s}}(x)>0\right\}\right|}{h_{1}}>\frac{1}{2 n M}>0 .
$$

If we choose $\eta_{n}=1 / 2 n M \in\left(0, \frac{1}{2}\right)$, then the definition of $M_{4}(A)$ is satisfied and the lemma is proved.

The following corollary is an immediate consequence of Theorems 4.1 and 1.2(c).

Corollary 4.4. If $f \in m \Sigma$ such that $f^{\mathrm{s}}$ is bounded, then $f^{\mathrm{s}} \in \mathfrak{\Re}_{4}(D(f))$.

Lemma 4.2 and Corollary 4.4 improve upon the original results of Kundu [6] which require $f$ to be continuous and $f^{\mathrm{s}} \in \mathscr{N}$.

5. Some examples. In this section several examples are presented which illustrate the relationship between the classes $\mathfrak{N}_{4}, \mathfrak{N}_{5}$, ordinary derivatives and symmetric derivatives.

EXAMPLE. There is a bounded symmetric derivative, $h \in \mathfrak{N}_{4}$, which is not a derivative.

For each integer $n$ let $I_{n}=\left[2^{-n}, 2^{-n+1}\right]$. Partition each $I_{n}, n>0$, into $2^{n}$ equal subintervals $I_{n}^{k}, k=1, \ldots, 2^{n}$. If we write $[a, b]=I_{n}^{k}$, for some $k$ and $n$, then we may define a function $g_{n}^{k}$ such that $g_{n}^{k} \equiv 0$ on $(a, b)^{c}$,

$$
g_{n}^{k}\left(a+\frac{b-a}{4}\right)=-1, \quad g_{n}^{k}\left(a+\frac{3(b-a)}{4}\right)=1
$$

and $g_{n}^{k}$ is piecewise linear. Using these functions we define

$$
f(x)= \begin{cases}1 / 2, & x=0, \\ \sum_{n=0}^{\infty} \sum_{k=1}^{2^{n}} g_{n}^{k}(x)-g_{n}^{k}(-x)+\chi_{(-x, 0)}(x), & x \neq 0 .\end{cases}
$$

(If $A$ is any set, then $\chi_{A}$ is its characteristic function.)

To show that $f \in \mathscr{N}_{4}$, let $\alpha \in \mathbf{R}$ and define $E=\{x: f(x)>\alpha\}$. If $\alpha \geqslant \frac{1}{2}$, then it is clear from the continuity of each $g_{n}^{k}$ that $E$ is open and, consequently, $E \in \Re_{4}$. So, we suppose $\alpha<\frac{1}{2}$. We may write

$$
E=\bigcup_{n=1}^{\infty}\left\{x: f(x) \geqslant \alpha+\left(\frac{1}{2}-\alpha\right) / n\right\} .
$$

From the definition of $f$ it follows that for each $n$ the set

$$
E_{n}=\left\{x: f(x) \geqslant \alpha+\frac{\frac{1}{2}-\alpha}{n}\right\}
$$


consists of the set $\{0\}$ and a sequence of disjoint closed intervals converging bilaterally to 0 . This makes it clear that each $E_{n}$, and therefore $E$, is an $F_{\sigma}$ set. We choose the sequence $\left\{E_{n}\right\}$ to be the sequence of closed sets in the definition of $\mathfrak{M}_{4}$. From above, we see that $E_{1}$ is closed and $E_{1} \subset E_{n}$ for each $n$.

Choose $c>0$ and let $r$ be the smallest integer such that $c<2^{r-3}$. Pick $\varepsilon(0, c) \in$ $\left(0,2^{-r}\right)$ and let $h$ and $h_{1}$ be positive numbers such that $h<c h_{1}$ and $h+h_{1}<\varepsilon(0, c)$. There exists an integer $s>r$ such that $h \in\left[2^{-s}, 2^{-s+1}\right)$.

We claim there exist integers $i$ and $j$ such that $I_{i}^{j} \subset\left(h, h+h_{1}\right)$. To see this, suppose not. Then $\left(h, h+h_{1}\right)$ can intersect at most two of the intervals $\left\{I_{n}^{k}\right\}$. Using the fact that $h<2^{-s+1}$, we see

$$
h_{1} \leqslant\left|I_{s}^{2^{s}}\right|+\left|I_{s-1}^{1}\right|=5\left(2^{-2 s}\right)<2^{-2 s+3} .
$$

This implies

$$
h / h_{1}>2^{-s} / 2^{-2 s+3}>2^{r-3}>c
$$

by the choice of $r$. This contradicts the choice of $h<c h_{1}$. Therefore, the claim is true.

Now, let $\left\{J_{1}, \ldots, J_{m}\right\}$ be the set of all intervals from $\left\{I_{n}^{k}\right\}$ such that $J_{i} \cap(h, h+$ $\left.h_{1}\right) \neq \varnothing$ and such that the $J_{i}$ are arranged in increasing distance from 0 . It is clear from above that $m \geqslant 3$. Thus, $h \in J_{1}, h+h_{1} \in J_{m}$ and $J_{i} \subset\left(h, h+h_{1}\right)$ for $2 \leqslant i \leqslant$ $m-1$. From the definition of $g_{n}^{k}$ we see that for $2 \leqslant i \leqslant m-1$,

$$
\left|E_{1} \cap J_{i} \cap\left(h, h+h_{1}\right)\right|=\left|E_{1} \cap J_{i}\right|=\left|J_{i}\right| / 4
$$

so that

$$
\left|E_{1} \cap \bigcup_{i=2}^{m-1} J_{i}\right|=\left|\bigcup_{i=2}^{m-1} J_{i}\right| / 4 .
$$

From the definition of the $I_{n}^{k}$ it follows that $\left|J_{1}\right| \leqslant\left|J_{2}\right|$ and $\left|J_{m}\right| \leqslant 4\left|J_{m-1}\right|$ so that

$$
\left|\bigcup_{i=1}^{m} J_{i}\right|=\left|J_{1}\right|+\left|\bigcup_{i=2}^{m-1} J_{i}\right|+\left|J_{m}\right| \leqslant 6\left|\bigcup_{i=2}^{m-1} J_{i}\right| .
$$

Statements (21) and (22) imply

$$
\frac{\left|E_{1} \cap\left(h, h+h_{1}\right)\right|}{h_{1}} \geqslant \frac{\left|E_{1} \cap \bigcup_{i=2}^{m-1} J_{i}\right|}{\left|\bigcup_{i=1}^{m} J_{i}\right|} \geqslant \frac{1}{24} \text {. }
$$

Choose $\eta$ such that $0<\eta<1 / 24$. Then (23) implies

$$
\frac{\left|E \cap\left(h, h+h_{1}\right)\right|}{h_{1}} \geqslant \frac{\left|E_{1} \cap\left(h, h+h_{1}\right)\right|}{h_{1}}>\eta .
$$

If $h$ and $h_{1}$ are chosen to be negative such that $h<c h_{1}$ and $h+h_{1}>-\varepsilon(0, c)$, then a similar argument may be used to show that

$$
\left|E \cap\left(h, h+h_{1}\right)\right|<-\eta h_{1} \text {. }
$$


Therefore, in the definition of the class $M_{4}\left(D\left(\mu_{f}\right)\right)$, if we let $\eta_{n}=\eta \in\left(0, \frac{1}{24}\right)$ for each $n$, then the definition is satisfied at $x=0$ with the set $E$. If $x \in E$ such that $x \neq 0$, then there is a neighborhood of $x$ contained in $E$. From this it is evident that the criteria for the definition of $M_{4}$ are satisfied at $x$. This argument shows $E \in M_{4}$.

If $E=\{x: f(x)<\alpha\}$, then it is similarly established that $E \in M_{4}$. Thus, $f \in \Re_{4}$.

Now let $F(x)=\int_{0}^{x} f(t) d t$. Since $f$ is continuous on $(-\infty, 0) \cup(0, \infty), F$ is differentiable with $F^{\prime}(x)=f(x)$ whenever $x \neq 0$. From the definition of $f$ and $F$, we also see

$$
\begin{aligned}
F^{\mathrm{s}}(0) & =\lim _{x \rightarrow 0} \frac{F(x)-F(-x)}{2 x}=\lim _{x \rightarrow 0} \frac{1}{2 x} \int_{0}^{x}(f(t)-f(-t)) d t \\
& =\lim _{x \rightarrow 0} \frac{1}{2 x} \int_{0}^{x} d t=f(0) .
\end{aligned}
$$

It follows that $F \in m \Sigma$ and $F^{s} \equiv f$.

Define the function

$$
g(x)= \begin{cases}1 / 2, & x=0, \\ \sum_{n=0}^{\infty} \sum_{k=1}^{2^{n}}-g_{n}^{k}(x)+g_{n}^{k}(-x)+\chi_{(-\infty, 0)}(x), & x \neq 0 .\end{cases}
$$

In the same manner as above, it can be established that $g \in \mathfrak{N}_{4}$ and $g$ is a symmetric derivative. But,

$$
f(x)+g(x)= \begin{cases}0, & x>0 \\ 1, & x=0 \\ 2, & x<0\end{cases}
$$

which violates the Darboux condition at $x=0$. Thus, either $f$ or $g$ is not a derivative. Let $h$ be the one which is not a derivative.

EXAMPLE. There exists an $f \in \Re_{5}$ which is a symmetric derivative, but not a derivative.

For each positive integer $n$ let $I_{n}=\left[2^{-n}, 2^{-n}+2^{-2 n}\right]$ and let $g_{n}$ be a nonnegative continuous function supported in $\left(2^{-n}, 2^{-n}+2^{-2 n}\right)$ with $\int_{I_{n}} g_{n}=2^{-n-1}$. Define

$$
g(x)=\sum_{n=1}^{\infty} g_{n}(x) \text { and } f(x)= \begin{cases}g(x), & x>0, \\ 0, & x=0, \\ -g(-x), & x<0 .\end{cases}
$$

Clearly $f$ is continuous on $(-\infty, 0) \cup(0, \infty)$. We must show that $f$ is approximately continuous at $x=0$. To do this, choose $c>0$, let $k$ be the largest positive integer such that $2^{-k} \geqslant c$ and let $N=\{x: f(x)=0\}$. Then

$$
|N \cap(-c, c)| \geqslant 2 c-2\left|\bigcup_{i=k}^{\infty} I_{i}\right|=2 c-2 \sum_{i=k}^{\infty} 2^{-2 i}=2 c-\frac{4}{3}\left(2^{-2 k+1}\right) .
$$

From this it is clear that

$$
\lim _{c \rightarrow 0} \frac{|N \cap(-c, c)|}{2 c}=1 .
$$


Therefore, 0 is a density point of $N$. Since $f(0)=0$, we see that $f$ is approximately continuous at $x=0$.

Let $F(x)=\int_{0}^{x} f(t) d t$. Then $F^{\prime}(x)$ exists and equals $f(x)$ when $x \neq 0$. Also,

$$
\begin{aligned}
F^{\mathrm{s}}(0) & =\lim _{h \rightarrow 0} \frac{F(h)-F(-h)}{2 h}=\lim _{h \rightarrow 0} \frac{\int_{0}^{h} f(t) d t-\int_{0}^{-h} f(t) d t}{2 h} \\
& =\lim _{h \rightarrow 0} \frac{\int_{0}^{h} g(t) d t-\int_{0}^{h} g(t) d t}{2 h}=0 .
\end{aligned}
$$

Therefore, $F \in m \Sigma$ with $F^{\mathrm{s}}=f$.

To see that $f$ is not a derivative, we first note that

$$
\begin{aligned}
D^{+} F(0) & \geqslant \lim _{n \rightarrow \infty} 2^{n} \int_{0}^{2^{-n}} f(t) d t \\
& =\lim _{n \rightarrow \infty} 2^{n} \sum_{m=n}^{\infty} 2^{-m-1}>1>F^{\mathrm{s}}(0)=0
\end{aligned}
$$

so $F^{\prime}(0)$ does not exist. Since $F$ is absolutely continuous it is clear that $\mu_{F}=F$. Suppose $G$ is an ordinary primitive for $f$. Then $G=\mu_{G},(G-F)^{\mathrm{s}} \equiv 0$ and Theorem 1.3 implies there is a $c \in \mathbf{R}$ such that $F(x)=G(x)+c$. But, this implies $F$ is differentiable, which is a contradiction. Therefore, $f$ is not an ordinary derivative.

\section{REFERENCES}

1. C. L. Belna, M. J. Evans and P. D. Humke, Symmetric and ordinary differentiation, Proc. Amer. Math. Soc. 72 (1978), 261-267.

2. A. Bruckner, Differentiation of real functions, Lecture Notes in Math., vol. 659, Springer-Verlag, Berlin, 1978.

3. M. J. Evans, A symmetric condition for monotonicity, Bull. Inst. Math. Acad. Sinica 6 (1978), 85-91.

4. K. Garg, On a new definition of derivative, Bull. Amer. Math. Soc. 82 (1976), 768-770.

5. A. Khintchine, Recherches sur la structure des fonctions mesurables, Fund. Math. 9 (1927), 212-279.

6. N. K. Kundu, On symmetric derivatives and on properties of Zahorski, Czechoslovak. Math. J. 26 (1976), 154-160.

7. L. Larson, The symmetric derivative, Trans. Amer. Math. Soc. 277 (1983), 589-599.

8. C. E. Weil, A property for certain derivatives, Indiana Univ. Math. J. 23 (1973), 527-536.

9. Z. Zahorski, Sur la premiere dériveé, Trans. Amer. Math. Soc. 69 (1950), 1-54.

Department of MAThematics, University of Louisville, LOUiSVille, KentUCKy 40292 